\title{
Estimulação Cerebral Profunda: Nova Fronteira no Tratamento das Doenças do Sistema Nervoso Central
}

\author{
Deep Brain Stimulation: New Frontier in the Treatment of CNS Disorders
}

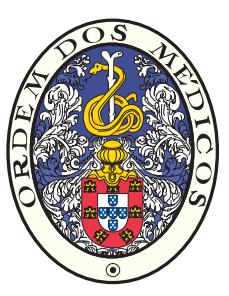

\author{
António GONÇALVES FERREIRA ${ }^{1}$ \\ Acta Med Port 2014 Sep-Oct;27(5):641-648
}

RESUMO

Introdução: O autor faz uma revisão das indicações atuais da estimulação cerebral profunda, dos seus desenvolvimentos e resultados, dos alvos e técnicas utilizados, dos principais neurocirurgiões e centros médicos envolvidos.

Material e Métodos: Esta revisão é baseada na experiência do grupo de Neurocirurgia Funcional do Hospital de Santa Maria (Centro Hospitalar Lisboa Norte) desde 1995 e na revisão bibliográfica dos artigos publicados sobre esta matéria desde 1980 indexados na PubMed.

Resultados e Discussão: São apresentadas e discutidas as aplicações da estimulação cerebral profunda nos diferentes domínios das neurociências funcionais: Doenças do Movimento, incluindo doença de Parkinson, distonias e tremores, síndrome de Gilles de la Tourette, dor crónica, epilepsia e psicocirurgia nas múltiplas áreas que esta compreende: perturbação obsessiva-compulsiva, depressão grave refratária, comportamento disruptivo com agressividade intratável, deterioração da memória e demência, perturbações do comportamento alimentar (anorexia nervosa, obesidade mórbida) e toxicodependências refratárias (álcool, opioides, cocaína).

Conclusões: Na maioria das situações clínicas referidas a estimulação cerebral profunda constitui um recurso terapêutico seguro e eficaz a ter em consideração quando as terapias habituais se mostram insuficientes; nos restantes casos há que reunir maior experiência até adotar o seu uso regular.

Palavras-chave: Estimulação Cerebral Profunda; Doença de Parkinson; Distonia; Tremor; Epilepsia; Psicocirurgia; Doenças do Sistema Nervoso Central.

\section{ABSTRACT}

Introduction: The author reviews the present indications of deep brain stimulation, its progress and outcomes, its targets and techniques, the main neurosurgeons and medical centers involved.

Material and Methods: This review is based on the experience of the Functional Neurosurgery group of the Hospital Santa Maria (Centro Hospitalar de Lisboa Norte) since 1995 and on the bibliography published since 1980 indexed on PubMed.

Results and Discussion: The deep brain stimulation applications in the different functional neurosciences fields are presented and discussed: Movement disorders, including Parkinson's disease, dystonia and tremor, Gilles de la Tourette, chronic pain, epilepsy and psychosurgery with its different aims: Obsessive-compulsive disorder, severe resistant depression, resistant aggressiveness with disruptive behavior, eating behavior disorders (anorexia nervosa, morbid obesity) and drug addiction (alcohol, opioids, cocaine).

Conclusions: In most of these clinical situations, deep brain stimulation is a safe and useful therapeutic resource that must be considered whenever the usual therapies are useless or not enough; in the other cases a greater experience is needed to make it a regular indication.

Keywords: Deep Brain Stimulation; Parkinson Disease; Dystonia; Tremor; Epilepsy; Psychosurgery; Central Nervous System Diseases.

\section{INTRODUÇÃO}

O tratamento das doenças do sistema nervoso central recebeu um enorme avanço com a introdução da Estimulação Cerebral Profunda (ECP). Esta modalidade terapêutica, desenvolvida a partir dos anos 80 do século XX e com crescimento exponencial neste século, é conhecida sobretudo pela sua designação em inglês - Deep Brain Stimulation (DBS) - e consiste na aplicação de corrente elétrica de baixa intensidade (0-10 volts), geralmente de alta frequência (100-250 hz) e largura de pulso variável (3-450 $\mu \mathrm{s})$, de forma contínua ou por ciclos intermitentes (cycling DBS) a estruturas nervosas do interior do encéfalo de forma muito seletiva (Fig. 1). Esta estimulação permite interferir com o funcionamento - Neuromodulação - dessas estruturas profundas (núcleos ou feixes com papel chave em diversas funções cerebrais) e aliviar sintomas de várias doenças do sistema nervoso central (SNC).
As primeiras e mais conhecidas indicações da ECP são as Doenças do Movimento - Doença de Parkinson (DP), Distonias, Tremor, síndrome de Gilles de la Tourette (GT) - mas a ECP tem tido igualmente indicação na terapêutica da Dor Crónica e, mais recentemente, em certas formas de Epilepsia e em algumas afeções psiquiátricas psico e farmacorresistentes: Perturbação Obsessiva-Compulsiva (POC), Depressão Major (DM), Comportamento Disruptivo com Agressividade Refratária. A título ainda experimental estão em curso os primeiros estudos respeitantes à sua utilização em tipos especiais de Demência, de Transtornos Alimentares (Obesidade, Anorexia) e de Toxicodependência.

A ECP é aplicada com elétrodos muito finos implantados nos alvos pretendidos, cuja localização tridimensional (3-D) e acesso cirúrgico exigem uma técnica especial -

1. Serviço de Neurocirurgia. Hospital de Santa Maria. Centro Hospitalar Lisboa Norte. Lisboa. Portugal.

Recebido: 03 de Março de 2014 - Aceite: 21 de Julho de 2014 | Copyright @ Ordem dos Médicos 2014 


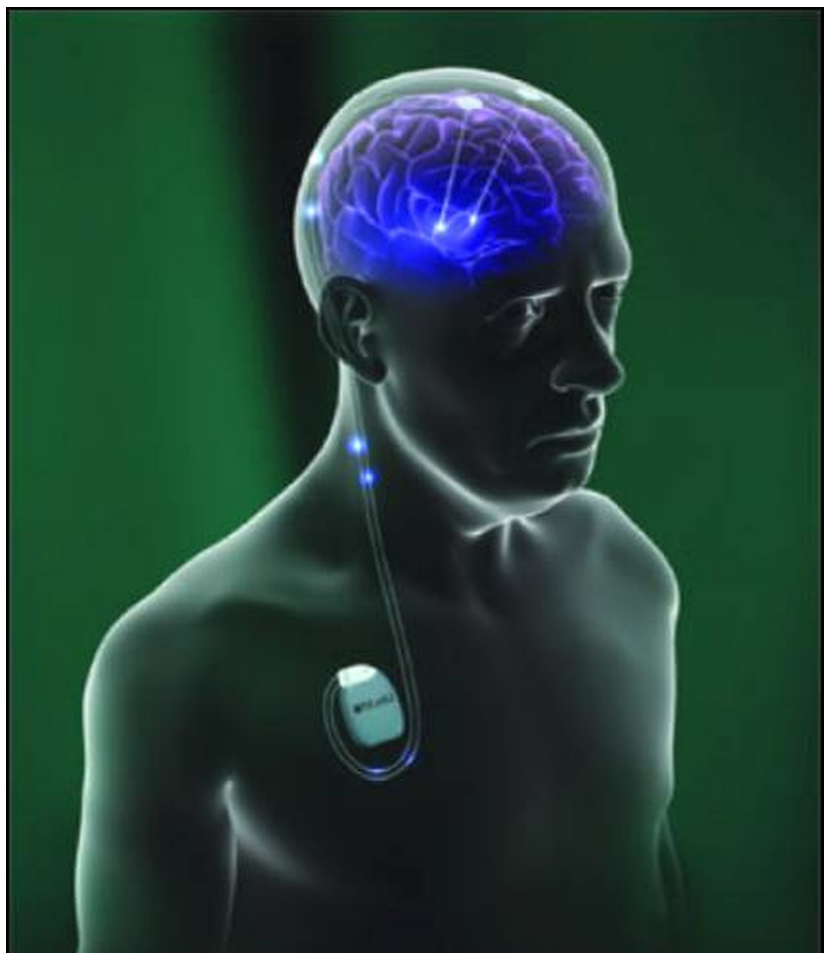

Figura 1 - Sistema de Estimulação Cerebral Profunda implantado (imagem gentilmente cedida por Medtronic ${ }^{\odot}$ )

Estereotaxia (Fig. 2) - que utiliza instrumentos sofisticados e tem uma elevada precisão (décimas de milímetro).

A implantação dos elétrodos (Fig. 3) é efetuada através de pequenos orifícios no crânio (trepanações, perfurações), em geral bilaterais. As trajetórias de acesso aos alvos são calculadas com recurso à moderna Imagiologia por Tomografia Computorizada e Ressonância Magnética (Neuronavegação) (Fig. 4). Além disso, a localização exata dos eléctrodos é auxiliada em muitos casos pelo registo eletrofisiológico profundo com microeléctrodos e confirmada por neurostimulação-teste efetuada durante a cirurgia. $O$ controlo clínico intraoperatório requer por isso que a maioria destes doentes (DP, Tremor, Dor, Psicocirurgia) se mantenham acordados e colaborantes durante a maior parte do procedimento, que pode durar muitas horas. A seleção dos parâmetros de estimulação, mono ou bipolar, tem ainda em conta que a extremidade de cada eléctrodo tem múltiplos contactos, o que possibilita regular também em cada caso a polaridade $+/ 0 /$ - dos contactos e escolher as suas múltiplas combinações. O eléctrodo é ligado através de um cabo subcutâneo a um neurostimulador (semelhante a um pace-maker) implantado a distância, geralmente numa loca subclavicular. A cirurgia para implantação de ECP exige assim uma vasta equipa multidisciplinar que inclui neurocirurgia, neurologia, neurofisiologia, neuroimagiologia, anestesia e também pessoal técnico e de enfermagem especializado; o estudo e o seguimento destes doentes envolve ainda, conforme os casos, a eletroencefalografia, a psiquiatria e a neuropsicologia.

Mercê desta sofisticada técnica (e tecnologia) a ECP
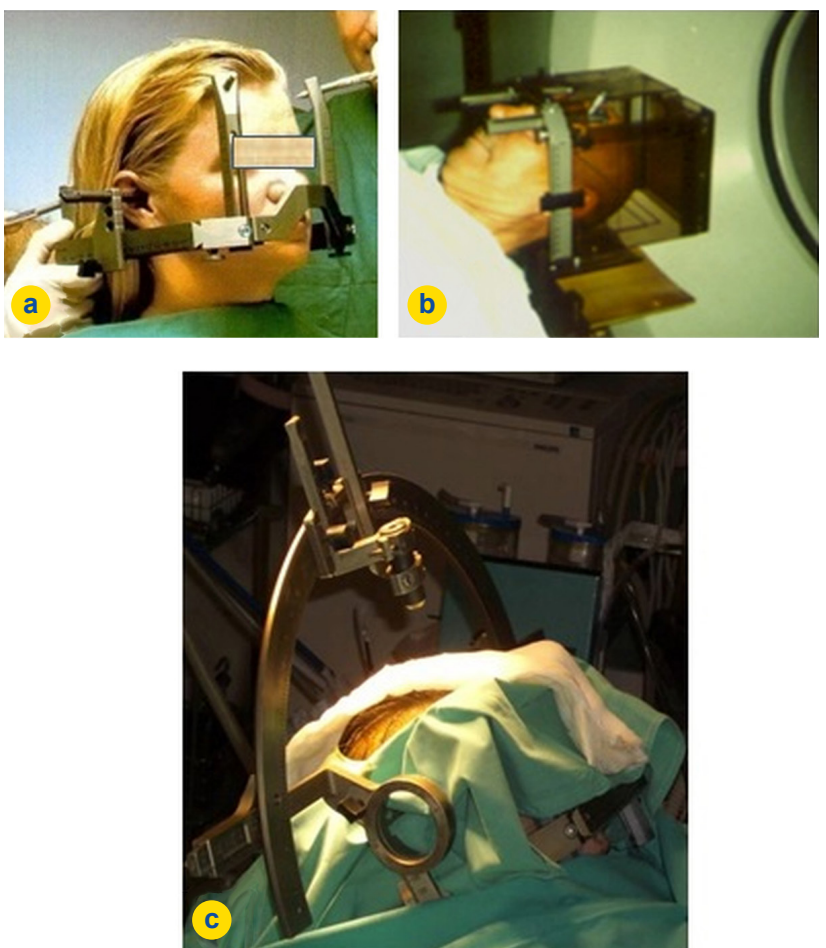

Figura 2 - Cirurgia Estereotáxica: a) Quadro estereotáxico fixado na cabeça do doente; b) TAC estereotáxico; c) Doente no bloco operatório com quadro e arco estereotáxicos colocados.

pode alterar e regular eletivamente o funcionamento das estruturas nervosas a que é aplicada, permitindo o controlo de manifestações clínicas e o alívio de diversas afeções, sobretudo quando outras formas de tratamento como a farmacoterapia e a psicoterapia se revelam insuficientes. Admite-se que os mecanismos de ação da ECP de alta frequência, apesar de não inteiramente esclarecidos, resultem num efeito predominantemente inibitório de neurónios e fibras nervosas (enquanto a ECP de baixa frequência é sobretudo excitatória). ${ }^{1,2} \mathrm{~A} E C P$, por ser uma técnica reversível e regulável, veio assim substituir com grande vantagem as técnicas destrutivas preexistentes usadas pela Neurocirurgia Funcional, cujas origens remontam à leucotomia de Egas Moniz, mesmo as mais seletivas (radiofrequência, criolesões, etc). E visa tratar também, não a causa das afeções, mas os mecanismos etiopatogénicos disfuncionais e as manifestações clínicas. Constitui assim uma revolução no armamentário terapêutico disponível para diversas doenças neurológicas e psiquiátricas, cujo espetro não pára de aumentar. Mais de 50000 procedimentos de ECP foram relatados até $2010,{ }^{3}$ mas perto do dobro terão sido tratados até agora, dos quais cerca de 500 em Portugal.

\section{MATERIAL E MÉTODOS}

Este estudo de revisão é baseado na experiência do grupo de Neurocirurgia Funcional do Hospital de Santa Maria - Centro Hospitalar de Lisboa Norte (HSM-CHLN) e na pesquisa da bibliografia publicada sobre este tema desde 1980 indexada na PubMed. Títulos pesquisados na PubMed: Deep Brain Stimulation, Movement Disorders, 

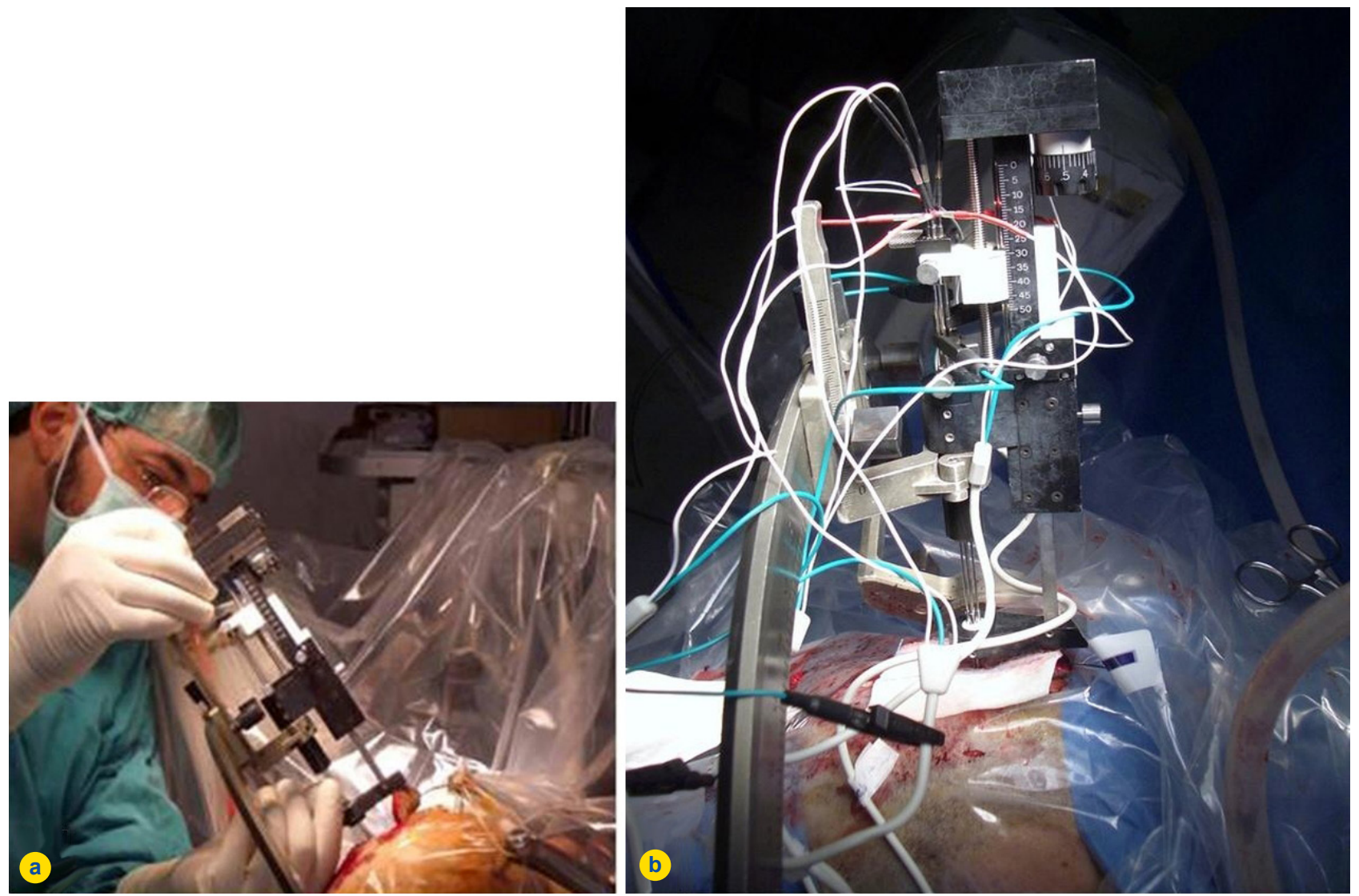

Figura 3 - Cirurgia Estereotáxica: a) Implantação dos elétrodos; b) Imagem em detalhe.
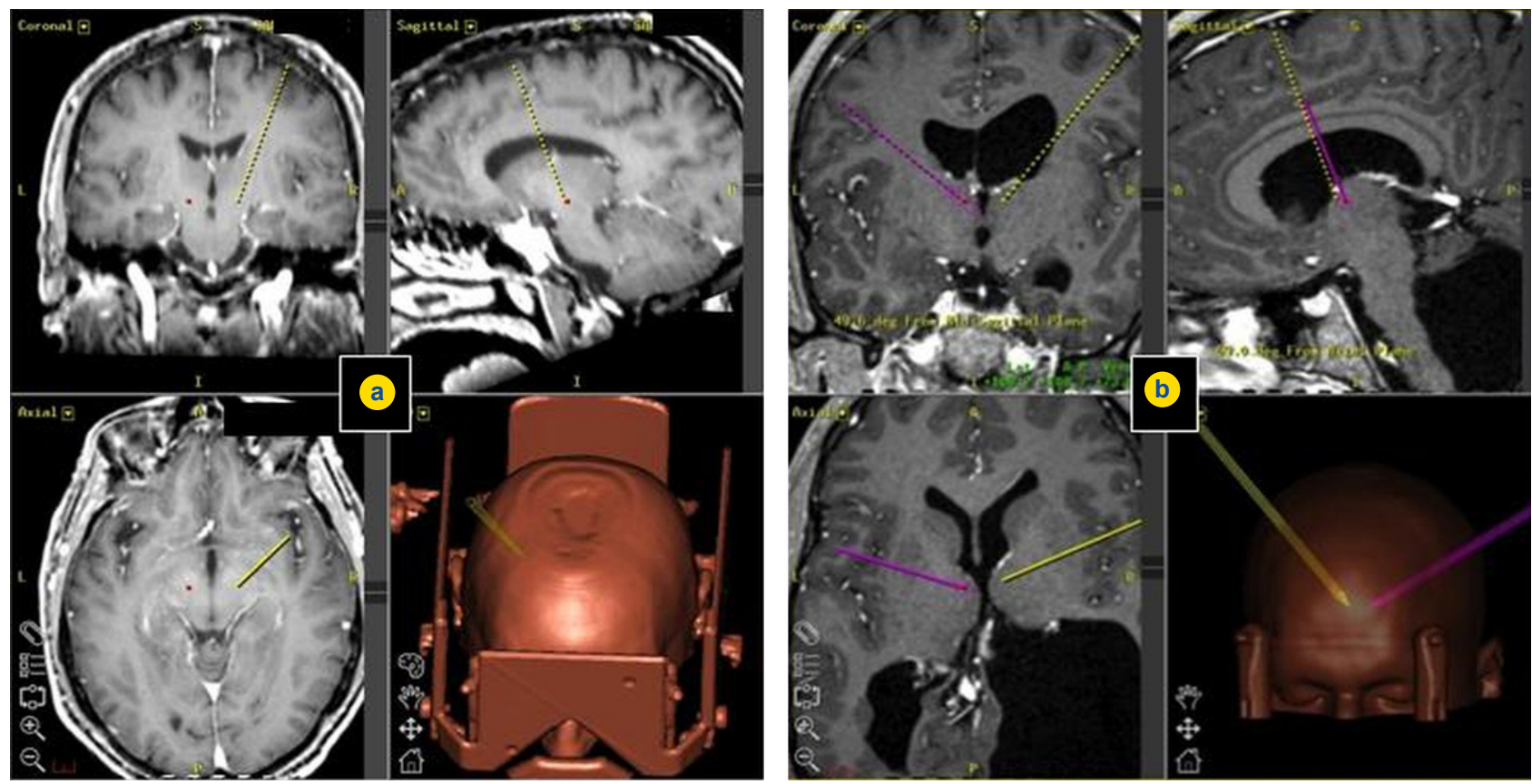

Figura 4 - Cirurgia Estereotáxica com Neuronavegação: planeamento de trajetórias de acesso aos alvos (imagens coronal, sagital, axial e reconstrução 3-D): a) tratamento da Doença de Parkinson; b) tratamento da Epilepsia

Parkinson Disease, Dystonia, Tremor, Chronic Pain, Epilepsy Surgery, Psychosurgery, Obsessive-Compulsive Disorder, Gilles de la Tourette, Major Depression, Aggressive and Disruptive Behavior, Eating Behavior Disorders (anorexia, obesity), Refractory Drug Addiction. Foram analisados 71 artigos de revistas e três capítulos de livros. 


\section{RESULTADOS E DISCUSSÃO}

Doença de Parkinson: A ECP foi iniciada em casos de tremor parkinsónico por Benabid em Grenoble em 1987 e anunciada nesse ano na Societé de Neurochirurgie de Langue Française em Paris. $O$ alvo da ECP era inicialmente $o$ núcleo ventral intermédio do tálamo (Vim), ${ }^{4}$ mas a partir de 1993 passou para o núcleo subtalâmico $(\mathrm{STN})^{5-8}$ por este se mostrar mais adequado ao tratamento da doença (Fig. 4). O STN tem sido desde então o alvo de eleição na DP em estádio avançado. O GPI é o alvo principal para ECP na distonia ou discinesia. ${ }^{9-17} \mathrm{O}$ Vim é atualmente o alvo preferencial para o tremor essencial ${ }^{13,18-21}$ ou outras formas de tremor não parkinsónico.

A melhor indicação para ECP no STN é a DP e o melhor critério de predição para a boa resposta a esta estimulação, em regra bilateral, é a resposta à medicação com levodopa.$^{22}$ Os parâmetros habituais de estimulação são 130 $\mathrm{Hz}$ de frequência, $60 \mu \mathrm{s}$ de largura de banda e 2,0-3,5V de intensidade, tendo o contacto ativo polaridade negativa. Os resultados a prazo são uma melhoria da ordem de $80 \%$ do tremor, $70 \%$ da rigidez e $50 \%$ da bradicinesia. $5,6,7,23$ A principal escala de avaliação da DP, a UPDRS (Unified Parkinson's Disease Rating Scale), melhora entre $55 \%$ e $70 \%{ }^{6,8}$ O maior benefício da ECP é a melhoria nas flutuações motoras e das discinésias provocadas pelos medicamentos anti-parkinsónicos. A dose diária de Levodopa pós-ECP é diminuída em média $40 \% .{ }^{6,8}$ Note-se que a demência e os défices cognitivos não melhoram com ECP (os problemas cognitivos e psiquiátricos podem até agravar-se com a ECP no STN), tal como a hipofonia e o gait freezing resistente à Levodopa. Para o controlo deste último tem sido também proposta a ECP do núcleo pedúnculo-pôntico. ${ }^{24} \mathrm{Em}$ geral, a idade varia de forma inversa com o bom resultado; na maioria dos centros só são submetidos a ECP doentes até aos 70 anos, mas este limite é cada vez mais flexível. A generalidade dos doentes operados têm história de 10-15 anos de doença; discute-se atualmente a indicação mais precoce para ECP na DP, questão objeto do estudo EARLYSTIM. ${ }^{25,26}$ A existência de um pace-maker cardíaco é habitualmente contraindicação para ECP, sobretudo com estimulação monopolar. Cerca de dois terços dos doentes com DP operados não sofrem efeitos adversos major, os efeitos secundários incluem apraxia, confusão transitória, hipomania e depressão pós-cirurgia numa minoria de doentes; infeções $(<5 \%)$ e hemorragias ( $<1 \%$ com défice permanente) são as complicações mais relevantes da $E C P ., 7 \mathrm{Em}$ Portugal foram até agora tratados com ECP perto de 400 doentes com DP, dos quais 150 no Hospital Santa Maria (HSM-CHLN), com resultados equiparados aos centros internacionais conhecidos, ${ }^{27}$ referidos acima: os doentes com DP operados no HSM tiveram em média uma melhoria motora (UPDRS-III) de $51,5 \%$, uma melhoria das atividades quotidianas (UPDRS-II) de $50 \%$ e do índice de Hoehn e Yahr (UPDRS-IV) de 63\%, com uma redução média diária de Levodopa de 62,8\%.

Distonia: A mais frequente indicação para ECP a se- guir à DP é a distonia e o principal alvo utilizado o GPi ventral-posterior ( $3 / 4$ a $4 / 5$ posterior) imediatamente por cima da fita óptica. ${ }^{14,15}$ Mas a distonia, ou melhor, as distonias são um grupo heterogéneo de perturbações com diferentes etiologias (primária ou secundária), distribuições anatómicas (generalizada, focal, segmentar) e idades de aparecimento. A ECP é muito eficaz nas distonias primárias, focais ou generalizadas, mas o seu resultado é mais variável nas secundárias. As formas mais frequentes de distonia focal ou segmentar são a distonia cervical (incluindo o torcicolo espástico), o blefarospasmo, a distonia oromandibular, a distonia craniofacial (síndrome de Meige) e a cãibra de escrivão. ${ }^{10-17} \mathrm{~A}$ ECP no GPi foi relatada em primeiro lugar na distonia cervical por Mundinger nos anos 70 , mas só teve uso generalizado no final do século $X X$. $O$ tratamento das distonias com ECP foi precedido de outros procedimentos invasivos sobretudo para as distonias focais, tais como desnervações periféricas, talamotomias e palidotomias estereotáxicas; as injeções repetidas de toxina botulínica, são muitas vezes eficazes nas alterações localizadas. Nas distonias primárias, generalizadas ou focais, a ECP tem em média $60 \%$ de bons resultados $(50-70 \%$ conforme os centros) enquanto as secundárias se quedam pelos 30 $60 \%$ consoante o tipo de distonia (piores resultados nas distonias pós-traumáticas e pós-paralisia cerebral).9.12-16 Os parâmetros da neurostimulação são análogos aos da DP. Nos doentes tratados com ECP no GPi, ao contrário dos DP tratados no STN, não se registaram efeitos secundários neurológicos a prazo, cognitivos ou depressivos; as complicações infecciosas e hemorrágicas são da mesma ordem. Em Portugal foram tratados cerca de 50 doentes distónicos (metade no HSM) com ECP no Gpi e os resultados são idênticos aos indicados acima: melhoria motora global de $45,1 \%$ (escala de Burke-Fahn-Marsden), com melhoria de $46,3 \%$ na subescala de incapacidade, valores que sobem respetivamente para $49,3 \%$ e $57 \%$ no respeitante às distonias primárias. Note-se que o tratamento farmacológico para as distonias é em geral sintomático. A gama etária para ECP nas distonias é mais larga que na DP e estende-se dos oito aos 75 anos.

Tremor: $\mathrm{O}$ tremor, sobretudo o tremor essencial, o mais prevalente $(0,4-4 \%$ da população), tem constituído indicação para ECP desde os finais dos anos 80 do século $X X$, quando se torna incapacitante e os restantes tratamentos falham. $4,18,28-30$ Mais frequente nos membros superiores, 0 alvo preferencial da ECP para o tremor é o núcleo ventral intermédio (Vim) do tálamo, em geral bilateral, em doentes sem DP ou distonia (nestes será o STN ou o GPi). Os resultados da ECP no Vim variam de centro para centro, dependem da etiologia, essencial ou secundária, de origem cerebelosa, rúbrica (tremor de Holmes) ou outra, e do tempo após a ECP; os bons resultados variam entre $60 \%$ e $90 \%$ a curto prazo, mas decaem com o tempo e situam-se a prazo nos $50-60 \%{ }^{19,28-30}$ Tratam-se adultos até aos $75-80$ anos de idade, com efeitos adversos reduzidos. ${ }^{4,19,30}$ 
Síndrome de Gilles de la Tourette (GT): A GT é uma síndrome neuropsiquiátrica hipercinética rara que compreende alterações do movimento e do comportamento, nomeadamente tiques motores e vocais (incluindo coprolália) e casos com automutilação. Dada a variabilidade da expressão clínica, os alvos para o tratamento têm sido muito diversos. Os primeiros casos de ECP na GT foram feitos por Visser-Vandewalle em 2003 no tálamo; 59 seguiram-se casos de estimulação do setor sensorio-motor do GPi e de estruturas límbicas (cápsula interna, núcleo accumbens). ${ }^{32,33}$ Foram tratados com ECP poucas dezenas de doentes. Os bons resultados, muito heterogéneos, atingem mais de $50 \%$ dos casos. ${ }^{34}$

Dor Crónica: $O$ tratamento da dor crónica resistente à medicação é outra indicação para ECP, em alternativa às técnicas ablativas e à neurostimulação medular ou periférica. ${ }^{35-40}$ Aziz et al (incluindo R. Vaz no Porto) trataram doentes a dores neuropáticas e de desaferentação; ${ }^{41}$ Franzini em Milão tratou casos de cefaleia crónica em salva (cluster) e de nevralgia disautonómica (SUNCT). ${ }^{37,38}$ Os alvos visados, muitas vezes unilaterais, encontram-se no tálamo (núcleo ventro-postero-lateral - VPL) ou no hipotálamo (póstero-medial e substância cinzenta periventricular - PVG). A generalidade dos doentes teve alívio da dor quantificável em 30 a $70 \%$, conforme o caso.

Epilepsia: A epilepsia farmacorresistente, sobretudo a epilepsia temporal (límbica) tem conhecido grandes progressos no tratamento microcirúrgico, ${ }^{42,43}$ mas quando este não está indicado, tem também sido objeto de tratamento com ECP. Os principais alvos (bilaterais) da ECP são os núcleos centro-mediano $(\mathrm{CMN})^{44,45}$ ou anterior (ANT) $)^{46}$ do tálamo, ou ainda o complexo amigdalo-hipocampo (A-H) ${ }^{47-49} \mathrm{~A}$ ECP no CMN, desenvolvida por Velasco et al nos anos 90 , visa a epilepsia generalizada e a epilepsia frontal; uma revisão recente da casuística do King's College de Londres e do Hospital La Princesa de Madrid ${ }^{44}$ mostra que a melhoria das crises ultrapassa $50 \%$, sendo mais evidente nas crises generalizadas. Desde 2000, o grupo do México ${ }^{48}$ e desde 2002 o grupo de Gent ${ }^{47,49}$ têm reunido experiência em ECP no A-H em doentes com epilepsia temporo-mesial não suscetíveis de cirurgia ablativa; registaram-se bons resultados na maioria dos casos, embora inferiores aos da remoção cirúrgica; esta técnica pode ser uma alternativa terapêutica para os doentes inoperáveis. Mais recentemente surgiu a ECP no ANT para casos de epilepsia focal envolvendo o sistema límbico, com um extenso estudo clínico multicêntrico feito nos EUA publicado em 2010 (estudo SANTE) ${ }^{46}$; este estudo prospetivo e aleatorizado reuniu 110 doentes cujo número de crises foi reduzido $>50 \%$ após dois anos e $>60 \%$ após quatro anos. Este foi o estudo mais robusto feito sobre ECP em epilepsia até agora. Decorre atualmente na Europa um estudo observacional dos casos submetidos a ECP no ANT (Fig. 4), que já reúne mais de 60 doentes (11 em Portugal, nove dos quais no HSM que co-lidera este estudo europeu); pretende-se estudar assim mais de 100 casos e esperam-se resultados conclusivos respeitantes à seleção de doentes, vias de acesso e parâmetros de estimulação. Os resultados preliminares revelam diminuição do número de crises como no estudo SANTE, mas com maior redução da intensidade das crises. Os principais efeitos colaterais são depressão (15\%) e alterações mnésicas (13\%), reversíveis. ${ }^{36}$ O ANT é, aliás, o único alvo de ECP para a epilepsia em que há experiência de muitos doentes tratados. Importa notar que a ECP para tratamento da epilepsia tem finalidade paliativa, devendo os doentes manter a medicação prévia; são poucos os casos de remissão completa das crises.

Psicocirurgia A Psicocirurgia, isto é a terapêutica cirúrgica de doenças psiquiátricas é, das indicações recentes para ECP, a que tem registado maior desenvolvimento. De facto, a possibilidade de melhorar substancialmente doentes mentais intratáveis com um procedimento focal, eletivo e reversível com técnica minimamente invasiva, fez reacender $\mathrm{o}$ interesse pela psicocirurgia; mesmo constituindo a ECP um último recurso. A Neuronavegação, surgida pouco depois da ECP, ao proporcionar a facilidade de guiar eléctrodos até qualquer alvo cerebral por imagiologia 3-D, potenciou a aplicação da ECP de forma precisa e segura. As perturbações psiquiátricas que mais têm beneficiado de ECP são a perturbação obsessiva-compulsiva ( $P O C)$, a depressão major (DM) refratária e casos de comportamento disruptivo com agressividade incontrolável. ${ }^{43}$

A POC é uma perturbação psiquiátrica de etiologia desconhecida com uma prevalência de cerca de $2 \%$ da população, ${ }^{51}$ que surge geralmente no adulto jovem e é caracterizada por pensamentos intrusivos (obsessões) e/ou actos ou comportamentos, ritualizados repetitivos (compulsões) muitas vezes associados a ansiedade e depressão. Cerca de $10 \%$ dos casos têm um curso crónico e refratário à psicoterapia e à farmacoterapia; são estes que têm potencial indicação para ECP. Esta modalidade terapêutica foi iniciada por Nutin em $1999^{51}$ e depois desenvolvida em múltiplos centros. A indicação para ECP nestes doentes, proposta por psiquiatras informados e experientes deve, como qualquer procedimento psicocirúrgico, ser aprovada por psiquiatras independentes e neurocirurgiões familiarizados com as técnicas envolvidas. Deve ter a chancela de comissões de ética e de supervisão, e em Portugal é obrigatória a aprovação pelo Conselho Nacional de Saúde Mental, tal como prescreve a Lei 36/98 de 24 de Julho (Lei de Saúde Mental). Note-se que a cirurgia é nestes doentes apenas uma parte do tratamento, uma vez que, sob ECP, devem prosseguir as restantes terapêuticas, medicação e psicoterapia. Foram até agora tratados com ECP cerca 50 doentes em todo o mundo (cinco no nosso país). A ECP na POC é geralmente bilateral, embora haja casos de estimulação unilateral com êxito. ${ }^{52,53}$ Os alvos escolhidos têm sido a cápsula interna (braço anterior), o núcleo da estria terminal e o núcleo accumbens (estruturas próximas envolvidas nos circuitos límbicos) $)^{31,51}$ ou ainda o STN ${ }^{54}$ ou o pedúnculo inferior do tálamo. ${ }^{55}$ Os resultados obtidos representam 
uma melhoria média de 50-70\%, embora muito variável de caso para caso. ${ }^{31}$ Os efeitos adversos neuropsiquiátricos são diminutos e as complicações análogas às das outras indicações para ECP.

A DM refratária à psicoterapia e à farmacoterapia é outra indicação para ECP. Os principais alvos, sempre dos dois lados, são estruturas que integram os circuitos de recompensa cerebral (sistema límbico) como o núcleo accumbens, desenvolvido por Schlaepfer ${ }^{56} \mathrm{e}$ a área cingular subcalosa 25 de Brodmann, por Lozano. ${ }^{23,57}$ Uma melhoria significativa da depressão observa-se em cerca de $60 \%$ dos casos, com uma redução igual ou superior a $50 \%$ na "Hamilton Disease Rating Scale for Depression". ${ }^{56,57}$ A correta seleção dos doentes é um dos aspetos difíceis nestes casos. Tal como nas outras indicações para psicocirurgia, a ECP é apenas mais um elemento para o tratamento destes doentes, mesmo se decisiva; as restantes terapêuticas mantêm um papel imprescindível. A experiência internacional neste campo ainda é limitada e dispersa, não ultrapassando umas dezenas de casos.

Uma outra indicação para ECP surgida recentemente e proposta pelo grupo de Franzini são os casos (raros) de comportamento violento e agressivo refratário ao tratamento, geralmente com atraso mental. ${ }^{38,58,60} \mathrm{O}$ alvo é o hipotálamo postero-medial (triângulo de Sano) bilateral. Seis de sete doentes tratados em Milão ${ }^{58} \mathrm{e}$ dois em Madrid ${ }^{60}$ tiveram resultado positivo.

Com carácter ainda experimental a ECP tem vindo a ser aplicada ao tratamento de casos de dismnésia por doença de Alzheimer ou DP. ${ }^{61,62}$ Os alvos, também bilaterais, são os pilares anteriores do fornix ${ }^{63,64}$ ou o núcleo basal de Meynert. ${ }^{65}$ Os resultados preliminares são positivos, mas os estudos estão ainda em curso.

Também alguns casos refratários de doenças do comportamento alimentar - Obesidade Mórbida e Anorexia Nervosa - têm sido objeto de tratamento experimental com ECP bilateral. No primeiro caso, o alvo escolhido foi o núcleo Accumbens ${ }^{66}$ ou a área cingular subcalosa 25 de Brodmann, este utilizado também na depressão pelo grupo de Toronto. ${ }^{23}$ Para a obesidade o alvo é o hipotálamo lateral. ${ }^{67}$ Aguardam-se resultados em breve.

Os primeiros casos de ECP em toxicodependentes reportam-se a doentes com alcoolismo crónico grave: o caso inicial foi tratado por ECP do núcleo accumbens em 2007 por Kuhn e Sturm. ${ }^{68} \mathrm{Em} 2012$ um outro grupo ${ }^{69}$ relatou mais cinco casos com mais de 10 anos de alcoolismo intratável, submetidos a ECP no mesmo alvo; dos cinco doentes, dois tiveram remissão completa do alcoolismo e um teve remissão parcial.

No que respeita à toxicodependência de drogas designadas 'duras', nomeadamente heroína ou cocaína, foram relatados recentemente alguns casos de ECP para tratamento de dependência de opióides (heroína): um caso em 2011 por Zhou, ${ }^{70}$ um caso em 2012 por Schuurman e Denys $^{71}$ e dois casos em 2014 por Kuhn e Sturm. ${ }^{72} \mathrm{O}$ alvo foi o núcleo accumbens nos quatro casos. Em todos se registou uma clara diminuição do consumo e do desejo de consumir.

Quanto à utilização de ECP para a dependência refratária a Cocaína, foi até agora tratado um único caso, pelo grupo do HSM em Lisboa. Dado o carácter pioneiro do caso, foi aplicado a este doente um protocolo especial de estudo e tratamento desenvolvido para avaliar o efeito terapêutico de ECP neste tipo de toxicodependência. É um protocolo de seguimento durante pelo menos dois anos, que incluiu um período de avaliação duplamente cega de seis meses e que cumpriu todas as exigências éticas e legais, nomeadamente a aprovação pela Comissão de Ética do HSM-CHLN e pelo Conselho Nacional de Saúde Mental. O alvo escoIhido, bilateral, foi baseado em estudos anatómicos prévios sobre o núcleo accumbens humano efetuados pela mesma equipa de investigação ${ }^{73,74} \mathrm{e}$ abrangeu a zona posterior deste núcleo, o núcleo da estria terminal e a porção mais profunda do braço anterior da cápsula interna. Este triplo alvo foi atingido, de cada lado, através da mesma trajetória vertical delineada com neuronavegação. Pretendeu-se assim interferir simultaneamente com algumas das principais estruturas dos circuitos cerebrais de recompensa envolvidas nesta doença. $O$ doente registou uma marcada redução do consumo e do desejo de consumo da droga, sem efeitos colaterais significativos além de um ganho ponderal evidente. Estes resultados foram já apresentados em congressos científicos internacionais e aguardam atualmente publicação in extenso.

\section{CONCLUSÕES}

A ECP é uma nova modalidade terapêutica, segura e eficaz, que constitui hoje uma importante alternativa para o tratamento de diversas doenças crónicas do SNC. Está bem estabelecido o seu papel na DP, Distonias e Tremor. Com carater mais limitado, tem também indicação reconhecida em certas formas de Dor Crónica, de Epilepsia e de perturbações psiquiátricas como a POC e a Depressão Major. É ainda uma técnica em expansão como se prova pelos múltiplos campos experimentais a que se tem alargado como indicação off label, nomeadamente a Demência, os Transtornos Alimentares e certas Dependências refratárias.

$\mathrm{Em}$ todos estes casos a ECP envolve tecnologia sofisticada e complexa, e exige uma equipa multidisciplinar experiente para que seja planificada, executada e dirigida com a necessária eficiência e segurança. Deverá, por isso, estar sediada em departamentos de Neurociências Clínicas eletivos, com recurso a todas as valências que envolve e que constituam verdadeiros Centros de Referência no âmbito de Hospitais Centrais Universitários.

\section{AGRADECIMENTOS}

O autor agradece a ajuda na preparação deste artigo aos colegas que integram os grupos de trabalho de cada uma das áreas de referência de Neurociências Funcionais do HSM-CHLN envolvidas:

Cirurgia das Doenças do Movimento: Maria Begonia Cattoni, Herculano de Carvalho, Miguel Coelho, Leonor Guedes, Mário Miguel Rosa, Joaquim Ferreira; 
Cirurgia da Epilepsia (ECP): Alexandre Campos, Carla Bentes, Ana Rita Peralta, Inês Cordeiro, Carlos Morgado, Ana Santos, Luís Câmara Pestana, José Pimentel;

Psicocirurgia: Alexandre Campos, Frederico Couto, Diogo Ferreira, Joana Teixeira;

Neuroanatomia: Lia Neto, Edson Oliveira.

O autor agradece ainda especialmente a colaboração do colega Alexandre Campos na edição do filme vídeo em Apêndice.

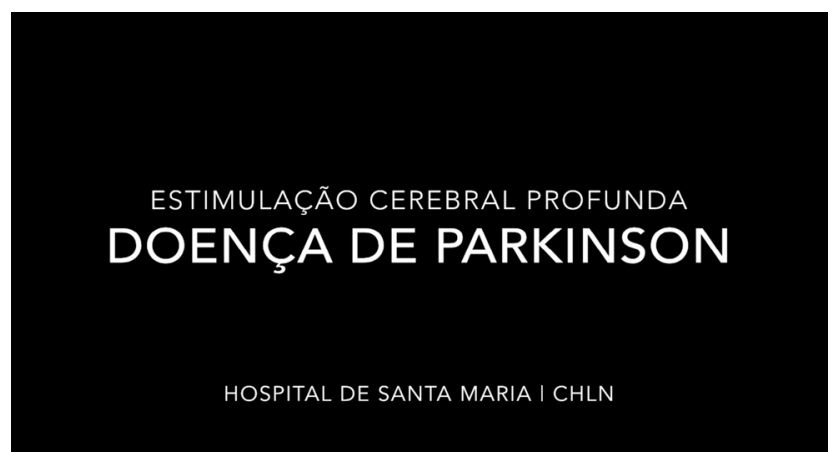

Apêndice - Vídeo Estimulação Cerebral Profunda. Hospital de Santa Maria/CHLN. [Link Vídeo].

\section{REFERÊNCIAS}

1. Mclntire C, Savasta M, Kerkerian-Le Goff L, Vitek JL. Uncovering the mechanisms of action of deep brain stimulation: activation, inhibition, or both. Clin Neurophysiol. 2004;115:1239-48.

2. Montgomery EB Jr, Gale JT. Mechanisms of action of deep brain stimulation (DBS). Neurosc Biobehav Revi. 2008;32:388-407.

3. Talan J. Deep brain stimulation. Washington: DANA Press; 2009.

4. Benabid AL, Pollak P, Gao D, Hoffmann D, Limousin P, Gay E, et al. Chronic electrical stimulation of the ventralis oralis nucleus of the thalamus as a treatment of movement disorders. J Neurosurg. 1996;84:203-14

5. Hariz MI, Rehncrona S, Quinn NP, Speelman JD, Wensing C. Multicentre Advanced Parkinson's Disease Deep Brain Stimulation Group: Multicentre study on deep brain stimulation in Parkinson's disease: an independent assessment of reported adverse effects at 4 years. Mov Disord. 2007;22:1886-70.

6. Kleiner-Fishman G, Herzog J, Fishman DN, Tamma F, Lyons KE, Pahwa $\mathrm{R}$, et al. Subthalamic nucleus deep brain stimulation: summary and meta-analysis of outcomes. Mov Disord. 2006;21:290-304.

7. Krack P, Batir A, Van Blercom N, Chabardes S, Fraix V, Ardouin C, et al. Five year follow-up of bilateral stimulation of the sub-thalamic nucleus in advanced Parkinsons's disease. N Engl J Med. 2003;349:1925-34.

8. Limousin P, Krack P, Lollak P, Ardouin C, Hoffmann D, Benabid $\mathrm{AL}$. Electrical stimulation of the subthalamic nucleus in advanced Parkinson's disease. N Engl J Med. 1998;339:1105-11.

9. Cappele $\mathrm{HH}$, Weigl R, Krauss JK. Bilateral pallidal stimulation for blepharospasm-oromandibular dystonia (Meige syndrome). Neurology. 2003;60:2017-8

10. Coubes P, Cif L, El Fertit H, Hemm S, Vayssiere N, Serrat S, et al. Electric stimulation of the globus pallidus internus in patients with primary generalized dystonia: long term results. J Neurosurg. 2004;101:189-94.

11. Krauss JK, Pohle T, Weber,S, Ozdoba C, Burgunder JM. Bilateral stimulation of the globus pallidus internus for treatment of cervical dystonia. Lancet. 1999;354:837-8.

12. Kupsch A, Benecke R, Muller J, Trottenberg T, Schneider GH, Poewe $\mathrm{W}$, et al. Deep brain stimulation in primary generalized or segmental dystonia. N Engl J Med. 2006;355:1978-90.

13. Mueller J, Skogseid IM, Benecke R, Kupsch A, Trottenberg T, Poewe W. Deep Brain Stimulation for Dystonia Study Group. Pallidal deep brain stimulation improves quality of life in segmental and generalized dystonias: results from a prospective, randomized sham-controled trial. Mov Disord. 2008;23:131-4.

14. Starr PA, Turner RS, Rau G, Lindsey N, Heath S, Volz M, et al.

\section{CONFLITOS DE INTERESSE}

O autor é membro do Medtronic Registry for Epilepsy (MORE) European Steering Committee que supervisiona a experiência obtida na Europa com ECP no Núcleo Anterior do Tálamo para tratamento da epilepsia.

\section{FONTES DE FINANCIAMENTO}

Este trabalho não recebeu qualquer contribuição, subsídio ou bolsa.
Microelectrode-guided implantation of deep brain stimulaters into the globus pallidus internus for dystonia: techniques, electrode locations and outcomes. J Neurosurg. 2006;104:488-501.

15. Tisch S, Zrinco L, Limousin P, Bhatia KP, Quinn N, Ashkan K, et al. Effect of electrode contact location on Clinical efficacy of pallidal deep brain stimulation in primary generalized dystonia. J Neurol Neurosurg Psychiat. 2007;78:1314-9.

16. Vidaillet M, Vercueil L, Houeto JL, Krystkowiak P, Benabid AL, Cornu P, et al. Bilateral deep brain stimulation of the globus pallidus in primary generalized dystonia. N Engl J Med. 2005;352:459-67.

17. Yianni J, Bain P, Giladi N, Auca M, Gregory R, Joint C, et al. Globus pallidus internus deep brain stimulation for dystonic conditions: a prospective audit. Mov Disord. 2003;18:436-42.

18. Andy OJ. Thalamic stimulation for control of movement disorders. Appl. Neurophysiol. 1983;46:107-11.

19. Bain P. The results of DBS for tremor. In: Bain P, Aziz T, Liu X, Nandi D, editors. Deep Brain Stimulation. Oxford: University Press; 2009. p.15767.

20. Houeto JL, Karachi C, Mallet L, Pillon B, Yelnik J, Mesnage V, et al. Tourette's syndrome and deep brain stimulation. J Neurol Neurosurg Psych. 2005;76:992-5.

21. Jiménez F, Nicolini H, Lozano AM, Piedimonte F, Salín R, Velasco F. Electrical stimulation of the inferior thalamic peduncle in the treatment of major depression and obsessive-compulsive disorders. World Neurosurg. 2013;80:S30.e17-25.

22. Welter ML, Houeto JL, Teznas du Montcel S, Mesnage V, Bonnet AM, Pillon B, et al. Clinical predictive factors of subthalamic stimulation in Parkinson's disease. Brain. 2002;125:575-83.

23. Lozano AM, Giacogge P, Hamani C, Rizvi SJ, Kennedy SH, Kolivakis TT. A multicenter pilot study of subcallosal cingulated area deep brain stimulation for treatment-resistant depression. J Neurosurg 2012;116:315-22.

24. Mazzone P, Lozano A, Stanzione P, Galati S, Scarnati E, Peppe A, et al. Implantation of human pedunculopontine nucleus: a safe and clinically relevant target in Parkinson's disease. Neuroreport . 2005;16:1877-81.

25. Deuschl G, Schupbach M, Knudsen K, Pinsker MO, Cornu P, Rau J, et al. Stimulation of the subthalamic nucleus at an earlier disease stage of Parkinson's disease: concept and standards of the EARLYSTIM study. Parkinsonism Relat Disord. 2013;19:56-61.

26. Scheupbach WM, Rau J, Knudsen K, Volkmann J, Krack P, Timmerman L, et al. Neurostimulation for Parkinson's disease with early motor complications. N Engl J Med. 2013;368:610-22. 
27. Albuquerque L, Coelho M, Martins M, Guedes LC, Rosa MM, Ferreira $\mathrm{JJ}$, et al. STN-DBS does not change emotion recognition in advanced Parkinson's disease. Parkinsonism Relat Disord. 2014;20:166-9.

28. Koch M, Mostert J, Heersema D, De Keyser J. Tremor in multiple sclerosis. J Neurol. 2007;254:133-45.

29. Kudo M, Goto S, Nishikawa S, Soyama N, Ushio Y. Bilateral thalamic stimulation for Holme's tremor caused by unilateral brainstem lesion. Mov Disord. 2001;16:170-4.

30. Sydow O, Thobois S, Alesch F, Speelman JD. Multicentre European study of Thalamic stimulation in essential tremor: a six year follow-up. J Neurol Neurosurg Psychiat. 2003;74:1387-91

31. Nuttin B, Gabriels L, Cosyns P, Meyerson B, Andréewitch S, Sunaert $S$, et al. Long-term electrical capsular stimulation in patients with obsessive-compulsive disorder. Neurosurgery. 2003;52:1263-74.

32. Hodaie M, Wennberg R, Dostrovsky JO, Lozano AM. Chronic anterior thalamus stimulation for intractable epilepsy. Epilepsia. 2002;43:603-8.

33. Kuhn J, Lenartz D, Mai JK, Huff W, Lee SH, Koulousakis A, et al. Deep brain stimulation of the nucleus accumbens and the internal capsule in therapeutically refractory Tourette syndrome. J Neurol. 2007;6:6.

34. Servello D, Porta M, Sassi M, Brambilla A, Robertson MM. Deep brain stimulation in 18 patients with severe Gilles de la Tourette syndrome refractory to treatment: the surgery and stimulation. J Neurol Neurosurg Psych. 2008;79:136-42.

35. Coffey RJ. Deep brain stimulation for chronic pain: the results of two multi-center trials and a structured review. Pain Med. 2001;2:183-92.

36. Coffey RJ, Hamani C, Lozano AM. Evidence base: neurostimulation for pain. In: Winn N, editor. Youmans Neurological Surgery. $6^{\text {th }}$ ed. Philadelphia: Elsevier; 2011. p. 1821-24.

37. Franzini A, Ferroli $P$, Leone M, Broggi G. Stimulation of the posterior hypothalamus for treatment of chronic intractable cluster headaches: the first reported series. Neurosurgery. 2003;52:1095-99.

38. Franzini A, Marras C, Tringali G, Leone M, Ferroli P, Bussone G, et al Chronic high frequency stimulation of the posteromedial hipothalamus in facial pain syndromes and behaviour disorders. Acta Neurochir Suppl. 2007; $97: 399-406$

39. Nasndi D, Smith H, Owen S, Joint C, Stein J, Aziz T. Periventricular grey stimulation versus motor cortex stimulation for post stroke neuropathic pain. J Clin Neurosci. 2002;9:557-61.

40. Rodrigues JP, Walters SE, Watson P, Stell R, Mastaglia FL. Globus pallidus stimulation in advanced Parkinson's disease. J Clin Neurosci. 2007;14:208-15.

41. Pereira EAC, Boccard SG, Linhares P, Chamadoira C, Rosas MJ, Abreu $P$, et al. Thalamic deep brain stimulation for neuropathic pain after amputation or brachial plexus avulsion. Neurosurg Focus. 2013;35: 1-11.

42. Gonçalves-Ferreira A, Campos A, Herculano-Carvalho M, Pimentel J, Bentes C, Peralta AR, et al. Amygdalohippocampotomy: surgical technique and clinical results. J Neurosurg. 2013;118:1107-13.

43. Pimentel J, Bentes C, Campos A, Gonçalves Ferreira A. Long-term and late seizure outcome after surgery for temporal lobe epilepsy. Epileptic Disord. 2010;12:54-8

44. Valentin A, Garcia Navarrete E, Chelvarajah R, Torres C, Navas M, Vico $L$, et al. Deep brain stimulation of the centromedian thalamic nucleus for the treatment of generalized and frontal epilepsies. Epilepsia. 2013;54:1823-33

45. Velasco $A L$, Velasco $F$, Velasco $M$, Jiménez $F$, Marquez I, Rise $M$ Electrical stimulation of the centromedian thalamic nucleus in control of seizures: long-term studies. Epilepsia. 1995;36:63-71.

46. Fisher R, Salanova V, Witt T, Worth R, Henry T, Gross R, et al. SANTE Study Group: Electrical stimulation of the anterior nucleus of thalamus for treatment of refractory epilepsy. Epilepsia. 2010;51:899-908.

47. Boon P, Vonck K, De Herdt V, Van Dycke A, Goethals M, Goossens L, et al. Deep brain stimulation in patients with refractory temporal lobe epilepsy. Epilepsia. 2007;48:1551-60.

48. Velasco AL, Velasco F, Velasco M, Jiménez F, Carrillo-Ruiz JD, Castro $\mathrm{G}$. The role of neuromodulation of the hippocampus in the treatment of intractable complex partial seizures of the temporal lobe. Acta Neurochir Suppl. 2007; $97: 329-32$

49. Vonck K, Boon P, Achten E, De Reuck J, Caemert J. Long-term amygdalohippocampal stimulation for refractory temporal lobe epilepsy. Ann Neurol. 2002;52:556-65.

50. Kuhn J, Grundler TO, Lenartz D, Sturm V, KlosterKotter J, Huff W Deep brain stimulation for psychiatric disorders. Dtsch Arztebl Int. 2010;107:105-13

51. Nuttin B, Cosyns P, Demeulemeester H, Gybels J, Meyerson. Electrical stimulation in anterior limbs of internal capsules in patients with obsessive-compulsive disorder. Lancet. 1999;354:1526.

52. Barcia JA, Reyes L, Arza R, Sacedo J, Avecillas J, Yañes R, et al. Deep brain stimulation for obsessive-compulsive disorder: is the site relevant? Stereotact Funct Neurosurg. 2014;99:31-6.

53. Huff N, Lenartz D, Schurmann M, Lee SH, Kuhn J, Koulousakis A, et al. Unilateral deep brain stimulation of the nucleus Accumbens in patients with treatment-resistant obsessive-compulsive disorder. Outcome after one year. Clin Neurol Neurosurg. 2010;112:137-43.

54. Chabardes S, Polosan M, Krack P, Bastin J, Krainik A, David O, et al Deep brain stimulation for obsessive-compulsive disorder: subthalamic nucleus target. World Neurosurg. 2013;80:S31.e1-8.

55. Israel M, Steiger H, Kollvakis T, McGregor L, Sadikot AF. Deep brain stimulation in the subgenual cingulated cortex for an intractable eating disorder. Biol Psychiatry. 2010;67:e.53-54

56. Bewernick BH, Kayser S, Sturm V, Schlaepfer TE. Long-term effects of nucleus Accumbens deep brain stimulation in treatment-resistant depression: evidence for sustained efficacy. Neuropsychopharmacology. 2012;37:1975-85

57. Mayberg HS, Lozano AM, Voon V, McNeely HE, Seminowicz D, Haman $C$, et al. Deep brain stimulation for treatment-resistant depression. Neuron. 2005;45:651-60.

58. Franzini A, Marras C, Ferroli P, Bugiani O, Broggi G. Stimulation of the posterior hypothalamus for medically intractable impulsive and violent behavior. Stereotact Funct Neurosurg. 2005;83:63-6.

59. Temel Y, Visser-Vanderwalle V. Surgery in Tourette syndrome. Mov Disord. 2004;19:159-60.

60. Torres CV, Sola RG, Pastor J, Pedrosa M, Navas M, Garcia-Navarrete $\mathrm{E}$, et al. Long-term results of posteromedial hypothalamic deep brain stimulation for patients with resistente agressiveness. J Neurosurg. 2013;119:277-87

61. Hamani C, McAndrews MP, Cohn M, Oh M, Zumsteg D, Shapiro CM et al. Memory enhancement induced by hypothalamic/fornix deep brain stimulation. Ann Neurol. 2008;63:119-23.

62. Hardenacke K, Shubina E, Buhrle CP, Zapf A, Lenartz D, Klosterkotter J. Deep brain stimulation as a tool for improving cognitive functioning in Alzheimer's dementia: a systematic review. Front Psych. 2013;4:1-11.

63. Laxton AW, Lozano AM. Deep brain stimulation for the treatment of Alzheimer disease and dementias. Word Neurosurg. 2013;80:S28.e1-8.

64. Laxton AW, Tang-Wai DF, McAndrews MP, Zumsteg D, Wennberg R, Keren $\mathrm{R}$, et al. A phase I trial of deep brain stimulation of memory circuits in Alzheimer's disease. Ann Neurol. 2010;68:521-34.

65. Fontaine D, Deudon A, Lemaire JJ, Razzouk M, Viau P, Darcouirt $\mathrm{J}$, et al. Symptomatic treatment of memory decline in Alzheimer's disease by deep brain stimulation: a feasibility study. J Alzheimer's Dis. 2013;34:315-23

66. Wu H, Van Dyck-Lippens PJ, Santegoeds R, Van Kuyck K, Gabriels L, Lin G, et al. Deep-brain stimulation for anorexia nervosa. World Neurosurg. 2013;S29.e1-10.

67. Whiting DM, Tomycz ND, Bailes J, De Jonge L, Lecoultre V, Wilent B, et al. Lateral hypothalamic area deep brain stimulation for refractory obesity: a pilot study with preliminary data on safety, body weight and energy metabolism. J Neurosurg. 2013;119: 53-6.

68. Kuhn J, Lenarz D, Huff W, Lee S, Koulousakis A, Klosterkoetter J, et al. Remission of alcohol dependency following deep brain stimulation of the nucleus accumbens: valuable therapeutic implications? J Neurol Neurosurg Psych. 2007;78:1152-3.

69. Voges J, Muller L, Bogerts B, Munte T, Heinze HJ. Deep brain stimulation surgery for alcohol addiction. World Neurosurg. 2013;80:S28.e21-31.

70. Zhou H, Xu J, Jiang J. Deep brain stimulation of nucleus accumbens on heroin-seeking behaviors: a case report. Biol Psychiatry. 2011; 69:e412 .

71. Valencia-Alfonso $C E$, Luigjes J, Smolders $R$, Cohen $M X$, Levar $N$ Mazaheri $A$, et al. Effective deep brain stimulation in heroin addiction: a case report with complementary intracranial electroencephalogram. Biol Psychiatry. 2012;71: e35-7.

72. Kuhn J, Moller M, Treppman JF, Bartsch C, Lenartz T, Gruendler OJ, et al. Deep brain stimulation of the nucleus accumbens and its usefulness in severe opioid addiction. Mol Psychiatry. 2014;19:145-6.

73. Neto L, Oliveira E, Correia F, Gonçalves Ferreira AJ. The human nucleus accumbens, where is it? A stereotactic anatomical and magnetic resonance imaging study. Neuromodulation. 2008;11:13-22.

74. Neto L, Neto D, Oliveira E, Mourato B, Campos A, Gonçalves Ferreira $A$, et al. Three dimensional anatomy of the human nucleus accumbens. Acta Neurochir. 2013;155:2389-98. 


\section{Estimulação Cerebral Profunda: Nova Fronteira no Tratamento das Doenças do Sistema Nervoso Central}

Acta Med Port 2014:27:641-648

Publicado pela Acta Médica Portuguesa, a Revista Científica da Ordem dos Médicos

Av. Almirante Gago Coutinho, 151

1749-084 Lisboa, Portugal.

Tel: +351218428215

E-mail: submissao@actamedicaportuguesa.com

www.actamedicaportuguesa.com

ISSN:0870-399X | e-ISSN: 1646-0758

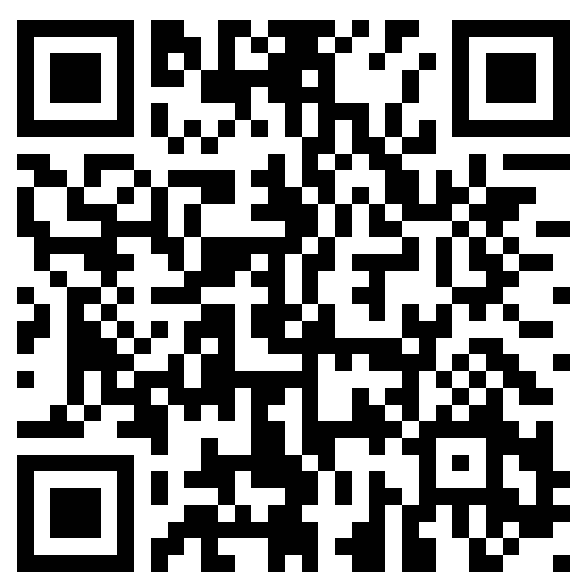

